\title{
Les biographies d'innovation comme vecteur de développement de la culture technique
}

\author{
Innovation biographies as a vector for technical culture development
}

\author{
Joelle Forest ${ }^{1}$, Lucie Dalibert ${ }^{1}$ \\ ${ }^{1}$ EA 4148 - S2HEP, Université de Lyon, lucie.dalibert@univ-lyon1.fr, joelle.forest@insa-Iyon.fr
}

\begin{abstract}
RÉSUMÉ. Nos sociétés contemporaines font face à un paradoxe de plus en plus criant. Alors que nous vivons dans un monde produit de plus en plus par la technique et que l'injonction à innover n'a jamais été aussi forte, la culture technique [CHO 17] peine à trouver sa place dans notre société. Or, la culture technique est non seulement un moyen de sortir les individus de leur aliénation [SIM 12] mais aussi indispensable si nous voulons chacun, en tant que citoyen, être à même de participer à la réflexion sur les grands défis contemporains. C'est précisément au projet de contribuer au développement d'une telle culture que souhaite contribuer le présent numéro spécial. II emprunte pour ce faire une perspective originale à savoir la biographie d'innovation.

ABSTRACT. Our contemporary societies face an increasingly glaring paradox. While we live in a world that is produced - more and more - by technology, and the demand for innovation has never been stronger, the technical culture [CHO 17] is struggling to find its place in our society. However, technical culture is not only a means of removing individuals from their alienation [SIM 12] but also indispensable if we, as citizens, want to be able to participate in the reflection on the great contemporary challenges. This special issue wishes to contribute to the development of such a culture. To this end, an original perspective is borrowed, namely the innovation biography.

MOTS-CLÉS. Biographie d'innovation, culture technique, histoire des techniques, innovation, processus de conception, sens de l'innovation.

KEYWORDS. Innovation biography, technical culture, history of technology, innovation, design process, sense- making.
\end{abstract}

Nous adhérons volontiers à l'idée de l'innovateur solitaire, voire de l'innovateur dans son garage à l'image de Steve Jobs créant son célèbre ordinateur Apple dans le garage de ses parents à Los Altos.

Une telle représentation n'est pas étrangère à la tendance de nos sociétés à mettre en scène l'innovateur et en faire une sorte de héro; tendance qui est particulièrement prégnante dans les biographies d'innovateurs [FOR 17] et perdure aujourd'hui via la propension qu'ont nos sociétés à célébrer chaque année des individus reconnus pour leurs idées prometteuses, à l'image des «Grands prix de l'innovation de la ville de Paris », du prix de l'innovation dans l'artisanat « The hands of innovation », du prix Vinci de l'innovation destiné à mettre en valeur une initiative menée sur le terrain par l'un de ses collaborateurs, ou du célèbre «Innovator of the year».

Si l'on peut comprendre le sens de cette dynamique (elle incarne et véhicule les vertus du courage, le goût de l'effort, met en scène le génie humain voire, sert de récit initiatique), force est cependant de constater que le focus mis sur l'individu conduit à donner une image tronquée du mode d'existence des innovations en laissant finalement dans l'ombre le processus de création et d'innovation. Aussi, l'ambition de ce numéro spécial vise-t-elle à opérer un renversement de perspective en mettant au premier plan l'innovation et en privilégiant la biographie de l'innovation à celle de l'innovateur.

Les biographies d'innovations apparaissent, en effet, comme un outil particulièrement opérant dans le cadre de la perspective artificialiste qui place au centre de sa réflexion le processus de conception des artefacts [FOR 18]. Elles contribuent au développement de connaissances sur le mode d'existence des innovations (en mettant en lumière l'épaisseur sociale de l'innovation, comment elle s'insère dans un entrelacs de contingences humaines, politiques, scientifiques, symboliques, etc.) et ce faisant au développement de la culture technique telle que définie par Marianne Chouteau, Joelle Forest et Céline Nguyen [CHO 17]. 
En effet, à suivre les auteurs, pour saisir mais aussi participer à la constitution de cette culture technique, il convient d'appréhender les objets techniques en prenant en compte trois niveaux :

1) celui des connaissances relatives à la structure et au fonctionnement de l'objet,

2) celui des savoirs et savoir-faire acquis et cultivés pour manier et utiliser l'objet, et,

3) celui des savoirs sur leur mode d'existence permettant de penser leur sens.

chacun d'entre eux renvoyant à un point de vue particulier sur l'objet (respectivement le point de vue du technicien, de l'usager et du citoyen) et ayant une portée agissante «Au premier niveau c'est elle [la culture technique] qui permet de garantir la robustesse de la solution développée, au niveau de l'usager c'est elle qui lui permet de comprendre l'usage et d'user de l'objet [...]. Au dernier niveau, c'est elle qui lui permet de prendre des décisions «politiques » [CHO $17: 6]$.

Comprendre précisément comment les biographies d'innovations contribuent au développement de la culture technique ? A quel niveau se situent lesdits enseignements ? Quelle est leur portée ? Tel est le fil conducteur des contributions rassemblées dans le présent numéro.

Comprendre comment est né le Micral ? C'est le projet auquel nous convie Loic Petitgirard dans son article Biographie de l'ordinateur R2E-Micral, ou comment faire exister un "micro-ordinateur 》 dans les années 1970s. On assiste alors à une véritable épopée dans laquelle l'auteur ne manque pas de mettre à jour les jeux des différents niveaux de culture technique. Impossible en effet de comprendre La genèse du Micral sans faire référence à l'importance de l'assise technologique des principaux protagonistes. Mais le Micral c'est aussi un « rêve »d'ingénieurs, pris entre les contraintes techniques du microprocesseur et les opportunités ouvertes par cette puce. C'est enfin un projet «politique »: la volonté d'ouvrir l'informatique et de démocratiser son utilisation.

Dans leur contribution, Marianne Chouteau, Joëlle Forest et Céline Nguyen présentent la biographie du procédé TEL de fibrage de la fibre de verre du groupe Saint Gobain. La biographie du TEL permet de voir les liens qui unissent cette innovation au contexte qui préside à sa genèse, que ce dernier soit considéré du point de vue des besoins, des technologies en place ou de la stratégie propre de Saint Gobain. Elle révèle également que la genèse du TEL fut loin d'être un long fleuve tranquille et matérialise des décisions prises par des acteurs clés. Si la biographie du TEL réactualise la thèse artificialiste selon laquelle la genèse d'une innovation ne peut être comprise qu'à partir d'une approche multi niveaux, elle conduit également à rompre avec l'idée de l'innovateur solitaire armé de la seule puissance de son cerveau et à accéder à la question du sens du TEL. A suivre les auteurs, ces trois enseignements constitutifs de l'approche biographique concourent à l'élaboration d'une culture technique qui devrait contribuer à la réflexivité du concepteur.

Dans leur contribution Emilie Hertig, Lucile Van Box Som et Dominique Vinck retracent l'histoire du projet Clean Space One, dont l'objet est de capturer et désorbiter des débris spaciaux. Chemin faisant les auteurs soulignent comment les biographies d'innovations enrichissent la culture technique des concepteurs en évitant de réduire l'invention à la recherche d'une solution à un problème, l'innovation à sa mise en œuvre et l'explication du succès par le génie d'individus hors norme. Loin de se réduire à l'affaire de quelques héros, Clean Space One mêle des collectifs qui explorent et entrelacent des questions de techniques, de bien commun, de financement, de normes internationales, de pédagogie et des rêves.

Dans leur article, Lucie Dalibert et Tjerk Timan construisent la biographie de la caméra Zepcam portée sur le corps par la police aux Pays-Bas en tant qu'outil de surveillance de la vie nocturne avec les outils de la théorie de .l'acteur-réseau [LAT 91] [AKR 92]. Cette biographie révèle un processus de conception chaotique et qu'au final c'est dans les pratiques des agents de police que le sens de la caméra portée au corps semble se stabiliser, du fait de ses fonctionnalités équivoques et de son décalage avec les usages des policiers. Ainsi, à travers la construction de la biographie de la Zepcam, l'article de Lucie Dalibert et Tjerk Timan participe non seulement au façonnement d'une culture 
technique mais aussi à la délimitation même de cette notion, en particulier dans leur insistance sur le fait que les finalités, les actions et finalement la signification de l'objet technologique n'est pas fixe mais se forme et se transforme au contact des différents acteurs qui s'en saisissent - ici les responsables politiques, les concepteurs et les utilisateurs - et ne constitue pas, en soi, une perspective à part.

Considérer l'objet technique non seulement en soi mais aussi dans son contexte pour construire sa biographie est une préoccupation partagée par Benjamin Bothereau qui, pour la réalisation de la biographie de la lanterne «à réverbères », distingue « deux moments biographiques » indispensables : le premier porte sur l'objet technique en lui-même et le second sur l'objet technique dans son milieu, la rue, qui est, depuis la fin du XVIIe siècle, marquée par un mouvement d'ordonnancement, de normalisation et de rationalisation. Ce deuxième moment biographique de la lanterne à réverbères matérialise et symbolise la maîtrise (et la police) de l'espace urbain au moyen de la technique. De la sorte, si la contribution précédente de Lucie Dalibert et Tjerk Timan. a trait à la façon dont les modes de surveillance de l'espace public urbain sont transformés par l'introduction de la caméra portée au corps, celle de Benjamin Bothereau montre comment, trois siècles auparavant, la lanterne à réverbères et plus généralement l'éclairage public a ouvert la voie et permis l'automatisation de la surveillance (policière) de l'espace nocturne parisien.

Dans son article, Amandine Gabriac revisite la photographie au XIXe siècle (1839-1901). En réalisant la biographie de cette innovation à partir de l'analyse des brevets d'invention conservés à l'Institut national de la propriété industrielle (INPI, France), celle-ci s'écarte des discours dominants qui associe tout progrès dans le domaine de la photographie à une personne et un nom (Niepce et Daguerre, Disdéri, Poitevin, etc.), lesquels deviennent célèbres. La relecture de cette innovation par un biais biographique révèle en effet que les inventions brevetées dans le domaine de la photographie ne sont jamais le fait d'une seule personne mais se nourrissent de connaissances, professions et réseaux pluriels. C'est ce constat qui conduit Amandine Gabriac a souligner comment cette relecture ouvre alors des perspectives sur ce que la photographie porte de culture technique autant que sur la culture technique de ceux qui y participent.

\section{Références :}

[AKR 92] AKRICH M., «The De-scription of Technical Objects », dans W. BIJKER et J. LAW, J. (dir.), Shaping Technology / Building Society : Studies in Sociotechnical Change, Cambridge, The MIT Press, 1992, pp. 205-224.

[CHO 17] Chouteau M., Forest J., NGuYen C., Quand la culture d'innovation fait écran à la culture technique, Technologie et innovation, volume 17(4), ISTE OpenScience, mis en ligne le 5 septembre 2017, https://www.openscience.fr/Quand-la-culture-d-innovation-fait-ecran-a-la-culture-technique, 2017.

[FOR 18] FOREST J., Rationalité créative et Innovation, ISTE Editions, février 2018.

[FOR 17] FOREST J., « Il n’y a pas de génie solitaire », La Recherche, Chronique Innovation, 530, p.37, 2017.

[LAT 91] LATOUR B., Nous n'avons jamais été modernes, Essai d'anthropologie symétrique, Paris, La Découverte, 1991.

[SIM 12] SIMONDON G., Du mode d'existence des objets techniques, Aubier, Paris, 2012. 